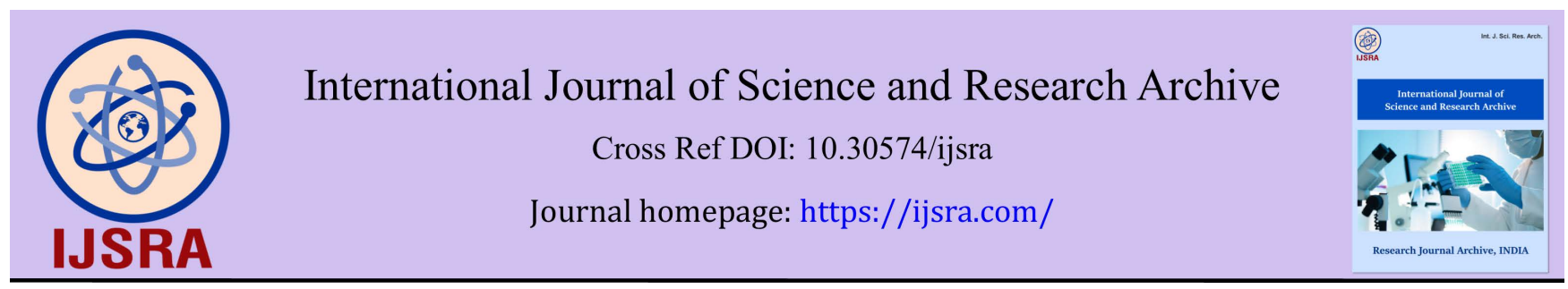

(REVIEW ARTICLE)

\title{
Artificial intelligence in advanced pharmacy
}

\author{
Tamanna Sharma ${ }^{1,}{ }^{*}$, Abhinav Mankoo ${ }^{1}$ and Vivek Sood ${ }^{2}$ \\ ${ }^{1}$ Pharmaceutical Sciences, Ggn Khalsa College of Pharmacy, Ludhiana, Punjab, India. \\ ${ }^{2}$ Cosmos Research lab. limited, Hambran Road, Ludhiana, Punjab, India.
}

International Journal of Science and Research Archive, 2021, 02(01), 047-054

Publication history: Received on 10 January 2021; revised on 02 Februay 2021; accepted on 04 Februay 2021

Article DOI: https://doi.org/10.30574/ijsra.2021.2.1.0301

\begin{abstract}
Artificial intelligence (AI) is Pharma's next frontier in life sciences. This article analyze about the recently techniques of AI that aims to imitate human intelligence functions i.e. with the help of Artificial intelligence \& Robots 'Automation become the result of Industrialization', driven by the need to increase productivity, to achieve consistent quality products \& to remove hazardous and heavy work from workers. Recent trends of AI in pharmacy are PAT, CFD, Pharmaceutical automation in research \& development which give detailed information about techniques that has already been used in healthcare such as inhaler designs, drug absorption \& dissolution and is disease focused. A robot for pharmaceutical applications has a bright future but with the rapidly aging population that urgently requires sophisticated medical devices \& newer drugs, robotics systems are increasingly adopted for improved productivity and efficiency to meet this growing demand. However, robots manufacturers face several challenges in their effort to establish themselves in pharmaceutical applications. AI with robotics in the life of mankind has several advantages \& disadvantages. Despite the increasingly rich AI literature from the drug discovery to care options AI techniques are used such as in ANN [artificial neural network], machine learning, AI in healthcare, AI in clinical practice. This research mainly concentrates around a few disease types: Cancer, Nervous system and cardiovascular diseases as they are life threatening. The future is always hard to predict, but it will be determined by AI as it would become the next frontier in pharmacy.
\end{abstract}

Keywords: Artificial intelligence [AI]; Chatbots; Computational fluid dynamics [CFD]; Robots, Artifical neural network; Applications of AI

\section{General Overview}

The current pharmaceutical industry's environment is plagued with expensive and lengthy drug discovery cycles coupled with pricing pressure by both Payers and Consumers. It is not enough to simply analyze drug discovery data but to remain competitive, Pharma must learn from the analytics.This is accomplished through yet another Disruptive technology -Artificial Intelligence [1]. AI may be defined in simple words that "It is the study of ideas which enable computers to do the things that make people seem intelligent". Artificial intelligence may be characterized as the scientific discipline pertaining to research studies, projects and applications that aim at supporting decision based medical tasks through knowledge or on computer based solutions that ultimately support and improve performance of a Human care provider [7].

The current drug discovery process needs to shift dramatically in order to meet needs of both society and patients in the 21st century. Pharmaceutical industry can accelerate innovation by using technological advancements. Pharmacy is one of the few top domains which can benefit the most from emergence of artificial intelligence, since its direct impact would be augmenting health, the epitome of evolution.

\footnotetext{
* Corresponding author: Tamanna Sharma

Pharmaceutical Sciences, Ggn Khalsa College of Pharmacy, Ludhiana, Punjab, India.
}

Copyright (C) 2021 Author(s) retain the copyright of this article. This article is published under the terms of the Creative Commons Attribution Liscense 4.0. 
Artificial intelligence aims to mimic human cognitive functions and objective is to bring a paradigm shift to healthcare data and rapid progress of analytical techniques.

\section{Artificial intelligence and Robotics}

Artificial intelligence and Robotics have a common root and a long history of interaction and scientific discussion.one might argue that not every machine is a Robot and certainly artificial intelligence is concerned also with virtual agents.

Artificial intelligence is a theory and robots are manufactured as hardware .The connection between these two is that the control of the robot is a software agents that reads data from these sensors decides what to do next and then directs the efforts to act in the physical world .It has wide application in robotics [2] . Furthermore, as patients become more engaged in their healthcare decision, they will turn to research possible medication options. Through target audience marketing, pharmaceutical companies can further assure the right information is presented at the right time to facilitate informed patent and provider discussions [1].

"It is time for connected Pharma"

However, progress is far from uniform and progress is likely to be "lumpy "at best .AI technology is well on its way to becoming ubiquitous and has huge scope, enhancing technology at many levels, leading to much better, faster patient outcomes.

\section{Pharmaceutical Automation}

With the help of Artificial intelligence Automation is the result of industrialization, driven by the need to increase productivity, to achieve consistent quality products and to remove hazardous and heavy work from workers. Innovations in technology now comprise the essential building blocks of Automation .Most Pharma players understand the benefit of adopting new technologies but there remains a persistent and troubling gap between strategy and organizations ability to adopt and deploy a data analytics working solution [1,3].

The adoption of AI allows for learning from real - time data.

- Identifying the right candidates for clinical trials.

- Processing real time patient feedback.

- Integrating data exchanges with partners.

- Distributors and caregivers.

There are just few examples on how to improve drug discovery outcomes, while aligning operational efficiencies to deliver better care to the patients, often getting the right medication to the right patient at right time is really about getting right information in front of healthcare provider. Armed with complete real-time drug insights, doctors are able to choose right prescription for the best possible outcome. Automation applications continue to grow with enabling technologies such as [4]:

- Wireless

- Nanotechnology

- Advance storage and memory

- Sensors and analyzers

- Advance software algorithms

- Artificial intelligence

\section{Recent trends}

\subsection{Pharmaceutical automation in Research and Development}

Artificial Intelligence is a more recent phenomenon. Though lab automation system have been around since the 90's ,it is only in recent years that it has taken off driver largely by laboratories devoted to activities such as high - throughput screening, combinatorial chemistry, automated clinical and analytical testing diagnostics and large scale biorepositories. Advancements in robotics and other technologies have made a fully automated library a reality [3]. The 
sheer throughput of measurements and data from the high speed and large quantity handling of samples demands a good automated information system. Example includes - LIMS (laboratory information management system).

The foundation for automation in pharmaceutical industry is clearly established .The functions that have already embraced automation will continue to prevail with incremental improvements from normal cycle of technology innovation [3].

\subsection{PAT (Process analytical technology)}

Another groundbreaking trend is in PAT. This plays an important role in helping pharmaceutical companies focus on continuous improvement and be more innovative to improve their manufacturing processes. This leads to improvement in product yields, Better utilization and less waste, translating into cost savings for patients .PAT is more than just instrumentation .It must be able to interface and collect data from various instruments and analyzers, has to perform complex multivariable calculations and modeling to understand critical process factors [3].

\subsection{Computational fluid dynamics}

Computational fluid dynamics is a design tool that allows designer of products to evaluate different designs rapidly and cost effectively.

Method works by using numerical methods to solve equations that govern fluid flow. A domain to be analyzed is first determined and split into thousands of small 3-D cells known as a computational mesh ,the fundamental equation (Navier -stokes) for fluid flow are solved automatically to arrive at an overall solution.

CFD can help overcome following issues, allowing the analysis of various drug delivery designs rapidly and economically, using a 3D model of human physiology.

- Traditionally ,Animal experimentation has been used to evaluate drug discovery systems but results are not seen as sufficiently reliable when transposed to humans, whose respiratory physiology ,for example, can differ greatly.This often leads to dramatically varying doses being released from same initial sample, making useful comparison problematic .

- An obligation exists under law to minimize experimentation on Animals; placing restrictions on the level of testing can be conducted.

\section{Applications of CFD}

Some of the current and possible applications of CFD in the development of drug delivery systems are revised below.

\subsection{Inhaler design}

Inhalation technology is extensively used for treating lung diseases such as asthma, cystic fibrosis. This method enable the rapid and easy administration of drugs and offers the ability to administer lowers dosages. Most of the drug absorption in the lung is across the alveolar epithelial. The rate of drug deposition depends on particle size and rate of inspiration. Dry powder inhalers and metered dose inhalers are two commonly available inhalers. MDI's containing CFC gases have been most prescribed type of inhalation system for past few years. However MDI's have two main weaknesses:

- Actuation of the aerosol.

- Low concentration of dose (Typically $10 \%$ of normal dose).

Pharmaceutical industry started researching inhaler design to improve factors such as:

- $\quad$ Ease of use.

- Efficiency of drug disposition.

- Reproducibility of drug dosage.

Drug delivery systems normally involve the transportation of additional phase material to the area being treated. These materials may be medicated particulate, liquid drops, a gaseous species or a mixture of these. A variety of established modeling methodologies is available to the CFD practitioner to scrutinize design variations. This allows the 
characterization of an effective device for the delivery of a specified dose concentration, dose variation, and particle dispersion. Equally, any cause of particle can be identified and subsequent design modifications can be tested to ensure and the problem is successfully addressed.

\subsection{Drug Absorption and Dissolution.}

Drugs can be administered via various different routes such as subcutaneously, rectally, orally, nasally and so on. CFD provides the ability to model such routes to predict dissolution and absorption of a drug in particular circumstances [5].

For example - Drugs intended for delivery via the respiratory tract are frequently micronized to create micro particles that are sufficiently small to be inhaled and targeted at a specific area of the lung or absorbed through alveolar epithelium and into the capillaries.

These particles have a high surface area and high charges, forming an unstable cohesive system. This means the particles could adhere to any surface on the way to the targeted region of the respiratory system. CFD can be used to trace the trajectories of a medication particle through nasal passage to its final deposition in the lung in order to ensure the necessary dosage is achieved.

\section{Pharmaceutical Applications}

Pharmaceutical industry can accelerate innovations by using technological advancements .The recent advancement that comes to mind would be artificial intelligence.AI can be of real help in analyzing the data and presenting results that would help out in decision making, saving human effort, time, and money thus help save lives [8].

\subsection{Drug Repositioning}

To identify the best available molecular starting points to Re -initiate a project with Re -Purposing a known drug or combination to test ,if it can treat another related or unrelated conditions based on its mechanism of action ,targets, Genomic or proteomic fingerprint[3].

\subsection{Alternative Indication Identification}

What are new promising indications for a particular class of inhibitors? By studying all the data pertaining to Indications and sorting them on quality, quantity and relevance in published research and trials [11].

\subsection{Epidemic outbreak prediction}

By using AI one can study history of epidemic outbreaks and the social media activity and also predict where and when an epidemic can affect with considerable accuracy.

Apart from the aforementioned use cases there are numerous others like!

- Personalizing the treatment

- Help build new tools for patients, physician etc.

\subsection{Robotics in pharmacy}

Robotics has a major role in dispensing system [5].

- $\quad$ Safety and quality - It leads to Reduction in dispensing errors and release staff from dispensary for direct patient care supporting [2,4].

- $\quad$ Financial-1. Reduced stock holding.

2. Improved stock rotation.

3. Reduction in Expired stock wastage.

- $\quad$ Process efficiencies - 1. Faster dispensing process to reduce patient waiting times.

2. Out of hour's activity. 


\section{Improved space utilization.}

- Reliability- The food and drug administration requires all medicines to be tracked and traced throughout the production process .Robots makes it easier for pharmaceutical companies to comply with these requirements. Along similar lines robots minimize accidents and waste materials [1].

- Production - with robots, throughput speed increase, this directly impacts production! Because robots have the ability to work at a constant speed without pausing for break, sleep, vacations, they have the potential to produce more than a human worker [10].

- Reduce chances of contamination -Removing people from the screening process reduces the risk of contamination and potential for dropped samples when handling them in laboratories. Robots perform these tasks much faster with more precision and accuracy.

- Increase efficiency -Robots can increase efficiency, which means price of drug itself become more competitive. When it comes to pharmaceutical production, people are not as efficient as robots, especially when they are wearing a protective suit [4].

\subsection{AI in Healthcare}

Before AI system can be deployed in health care applications, they need to be trained through data that are generated from the clinical activities such as screening, diagnosis, treatment and so on.

Specifically in the diagnosis stage, a substantial proportion of AI literature analyzes data from diagnosis imaging, Genetic testing and electrodiagnosis [6].

\subsection{Disease focus}

Despite the increasingly rich AI literature in healthcare, the research mainly concentrates around a few disease types: Cancer, Nervous system and Cardiovascular diseases.

- Cancer - Somashekharet et. al, demonstrated that the IBM Watson for oncology would be a reliable AI system for assisting the diagnosis of cancer through a double blinded validation study.

- Neurology- Bouton et.al developed an AI system to restore the control of movements in patients with quadriplegia.

- Farina et .al, tested the power of an offline man/ machine interface that uses the discharge timings of spinal motor neurons to upper -limb prostheses.

- Cardiology - Dilsizian and Siegel discussed the potential application of the AI system to diagnose the heart disease through Cardiac image. The concentration around this disease is not completely unexpected [9].

All three diseases are leading cause of death, therefore, early diagnosis are crucial to prevent deterioration of patients health status.

\subsection{AI in various techniques}

- Artificial Neural Network- It is the most interesting technique of artificial intelligence and is most commonly used in medicine. It is a powerful technique which includes Non-linear Mapping which is a mathematical system that stimulates biological neural networks .It models the pattern recognition capabilities of the neural networks of the brain.ANN can recognize patterns from complex sets of analytical data, they become very useful in data analysis of pharmacological research due to their ability to recognize even non-linear relationships from noisy data [7].

\subsubsection{Applications include-}

- $\quad$ Drug modeling

- Dosage design

- Protein structure

- Function prediction

- Pharmacokinetic and pharmacodynamic modeling

- In-vitro and In-vivo correlation 


\subsubsection{Machine learning}

Machine learning constructs data analytical algorithms to extract features from data. Input to machine learning algorithm 'traits' and sometimes medical outcomes of interest, which include Age, Gender, Disease history, Gene expression, clinical symptoms and medication [6].

\subsubsection{Deep learning}

Deep learning is a modern extension of the classical neural network technique .One can view deep learning as a neural network with many layers. Rapid development of modern computing enables deep learning to build up neural network with a large number of layers, which is infeasible for classical neural network.

\subsection{AI in clinical practice}

A major application of AI in healthcare is collecting, storing, normalizing and tracing data. Deep genomics aims to identify patterns in large data sets of genetic information and medical records, looking for mutations and linkages to the disease [11]. Working on a new generation of computational technologies that can cell physicians what will happen within a cell when DNA is altered by genetic variation, whether Natural or therapeutic.

Regarding the development of pharmaceuticals, Clinical trials take sometimes more than a decade and cost billions of dollars $[9,10]$.

\section{Advantages}

> Artificial intelligence presents the pharmaceutical industry with the opportunity to solve problems previously unsolvable with simple data analysis [8].

$>\mathrm{AI}$ is able to perform specific tasks and more accurately thereby reducing cost while increasing productivity.

$>$ AI offers valuable insights that will dramatically improve the outcomes of clinical trials.

$>$ Deep learning about Market dynamic, Customer behavior and their interplay.

$>$ Matching unmet customer needs with enhanced and differentiated value offerings - both Tangible and Intangible.

$>$ It improves the performance of antivirus detection systems and promotes production of new artificial intelligence algorithm.

$>$ It also helps in terms of the industry's selection of patient for clinical trials and enables companies to identify any issues with compounds much earlier when it comes to efficacy and safety.

> AI would have a low error rate compared to Humans, if coded properly. They would have incredible precision, Accuracy and speed.

$>$ Robotic radio surgery and other types of surgery in the future, can achieve precision that Humans can't.

$>\mathrm{AI}$ is reinventing drug discovery by using deep learning and natural language processing to understand and analyze vast quantities of bioscience information $[7,8]$.

\section{Disadvantages}

- AI mainly lacks in Human touch, since it doesn't have capacity to think, it can only function according to programs.

- It has the efficiency to corrupt younger generation.

- Can be changed foremost to mass scale destruction.

- If robots, starts to switch humans in all fields, it will finally lead to unemployment.

- Can cost a lot of money and time to build, repair and rebuild.

- Machines can easily lead to destruction, input in the wrong hands. That is, at least a fear of many humans.

- As seen partially with smartphones and other technologies already humans are dependent on AI and lose their mental capacities.

- $\mathrm{AI}$ as robots can supercede humans, enslaving us.

\section{Current challenges}

Healthcare's biggest challenge is its historical silted structure resulting from decade - old legacy systems. 
Pharmaceutical companies are further challenged by developing new drugs with greater efficacy and minimal adverse effects and to do so in an environment that results in greater development, success rates lower discovery costs and more direct access to patients in need[6].

- Rising customer expectations - The commercial environment is getting harsher, as healthcare payers impose new cost constraints on healthcare providers and scrutinize the value medicines offer much more carefully. They want new therapies that are clinically and economically better than the existing alternatives, together with hard, real world outcomes data to back any claims about medicine superiority [7].

- Poor scientific productivity- Pharma's output has remain at a stable level for the past decade .Using the same discovering and developing processes, there's little reason to think it's productivity will suddenly sore.

- Cultural sclerosis- The prevailing management culture, mental models and strategies on which relies are the same on it's traditionally relied on, even though they have been eclipsed by new ways of doing business.

These challenges are offset by promising advances in the collection and analysis of healthcare data coupled with machine learning capabilities. In the healthcare and pharmaceutical industry ,data continues to grow exponentially as a result of the R and D process itself, patients EHR's, feedback provided by caregivers[6,7]. Effectively utilizing these data points will help pharmaceutical companies better identify new potential drug candidates, develop them effectively, and ultimately getting new medications approved and Reimbursed more quickly.

\section{Future Directions}

\section{AI is Pharma's next frontier in life sciences.}

- Research and development- Pharmaceutical companies have need to develop portfolio's and in doing so must mitigate the risk by ensuring the appropriate allocation of $\mathrm{R}$ and $\mathrm{D}$ funds to enable decision making[1,6].

a. By increasing likelihood of successful drug discoveries.

b. Generate significant revenue growth.

c. To derive benefits from an integrated $\mathrm{R}$ and $\mathrm{D}$ i.e. manufacturing, sales and marketing environment.

- Clinical Trial Research- Clinical trials with AI have the potential to transform clinical trials, improve quality and safety of life enhancing therapies. It can predict drug activity with much higher precision than other methods. Clinical trials takes too much time and are very expensive, takes about 15 years to get a market ready product. The odd are not good, current drug discovery process is become less sustainable [11].

- Artificial Intelligence Powered Bots- Pharmaceutical companies in future be able to create bots for physicians in same way as they use Apps [7].

\section{For example}

$\checkmark \quad$ For a particular disease, bot that answers all types of patient queries about a specific disease. Educating patients by this is a specific treatment option that is launching soon.

$\checkmark$ For a particular treatment, Chatbots are developed, that allow patients or doctors to get opportunity for all relevant information about brand they require prescribing or starting treatment.

$\checkmark$ Once a treatment option is prescribed,now different kind of Chatbots can help with patient adherence .It can inform patients potential of side effects and how to react, provide reminder of when treatment should be administered and also to prevent video on how to properly administer dose and treatment

\section{Conclusion}

With the advanced current challenges and futuristic direction, AI would be beneficial to the world from finding a pharmacore for drug discovery \& development to healthcare like ANN, CFD \& Robotics. Artificial Intelligence offers insights generated from real-world data to provide more accurate information on patients and expected outcomes. So the Artificial Intelligence offers a compelling opportunity for pharmaceutical organizations i.e. working on a new generation of computational technologies that can tell physicians what will happen within a cell when DNA is altered by genetic variation. AI is reinventing drug discovery by using deep learning and natural language processing to understand and analyze vast quantities of bioscience information. 


\section{References}

[1] Russell S, Dewey D, Tegmark M. Research priorities for robust and beneficial artificial intelligence. Ai Magazine. 2015 Dec 31;36(4):105-14.

[2] Lakshmi Teja T, Keerthi P, Debarshi Datta NB. Recent trends in the usage of robotics in pharmacy.

[3] Yussupova N, Kovács G, Boyko M, Bogdanova D. Models and methods for quality management based on artificial intelligence applications. Acta Polytechnica Hungarica. 2016 Mar;13(3):45-60

[4] Brady M. Artificial intelligence and robotics. In Robotics and Artificial Intelligence 1984 (pp. 47-63). Springer, Berlin, Heidelberg.

[5] Mesko B. The role of artificial intelligence in precision medicine.

[6] Jiang F, Jiang Y, Zhi H, Dong Y, Li H, Ma S, Wang Y, Dong Q, Shen H, Wang Y. Artificial intelligence in healthcare: past, present and future. Stroke and vascular neurology. 2017 Dec 1;2(4).

[7] Patil DP. Emotion in artificial intelligence and its life research to facing troubles. International Journal of Research in Computer Applications and Robotics. 2016 Apr.

[8] Zhang Y, Balochian S, Agarwal P, Bhatnagar V, Housheya OJ. Artificial intelligence and its applications 2014.

[9] Dilsizian SE, Siegel EL. Artificial intelligence in medicine and cardiac imaging: harnessing big data and advanced computing to provide personalized medical diagnosis and treatment. Current cardiology reports. 2014 Jan 1;16(1):441.

[10] Neill DB. Using artificial intelligence to improve hospital inpatient care. IEEE Intelligent Systems. 2013 Jun 27;28(2):92-5.

[11] Roff HM. Advancing human security through artificial intelligence. Chatham House; 2017 May. 